\title{
Antitumor effect of the vascular-disrupting agent ZD6126 in a murine renal cell carcinoma model
}

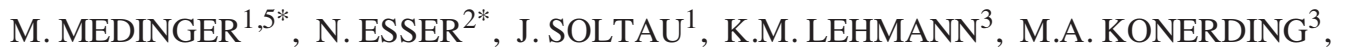 \\ T. WOLLOSCHECK ${ }^{3}$, A.J. RYAN ${ }^{4}$ and J. DREVS ${ }^{1}$ \\ ${ }^{1}$ Department of Medical Oncology, Tumor Biology Center at the Albert Ludwigs University; \\ ${ }^{2}$ ProQinase GmbH, Freiburg; ${ }^{3}$ Institute Functional and Clinical Anatomy, University Medical Center, \\ Johannes Gutenberg-University, Mainz, Germany; ${ }^{4}$ AstraZeneca, Alderley Park, Macclesfield, Cheshire, UK
}

Received September 29, 2010; Accepted November 3, 2010

DOI: $10.3892 /$ ijo.2010.867

\begin{abstract}
ZD6126 is a vascular-disrupting agent that affects the endothelial tubulin cytoskeleton causing selective occlusion of tumor vasculature and extensive tumor cell necrosis. The present study evaluated the antitumor and antivascular activities of ZD6126 in the clinically relevant murine renal cell carcinoma (RENCA) model and also evaluated biological response to therapy using color Doppler imaging as biomarker. Mice were implanted with RENCA tumor cells (day 0 ) and established tumors were treated with ZD6126 (100 mg/kg i.p.) or vehicle with repeated intermittent doses on day 10, 14 and 18. ZD6126 treatment led to a significant reduction in tumor size and was associated with extensive tumor necrosis and a reduction in tumor blood flow versus controls. MVD increased with intermittent treatment (day 10, 14 and 18). In an additional study, animals were treated at day 19 and quantitative three-dimensional microvascular corrosion casting was performed to enable detailed assessment of the tumor vascular architecture. Corrosion casting showed that tumor vessel architecture is affected by treatment, whereas pre-existing vessels in control tissues are practically not affected. Inter-vessel and inter-branch distances as well as vessel diameters are influenced by treatment. In conclusion, ZD6126 showed potent antitumor efficacy in the RENCA model and our data suggest that decrease in tumor blood flow may be a useful surrogate marker of treatment effect.
\end{abstract}

\section{Introduction}

New blood vessel formation (angiogenesis) is fundamental to tumor growth and spread (1). The importance of the angiogenic

Correspondence to: ${ }^{5}$ Present address: Dr Michael Medinger, University Hospital Basel, Hematology, Petersgraben 4, 4031 Basel, Switzerland

E-mail: medingerm@uhbs.ch

${ }^{*}$ Contributed equally

Key words: angiogenesis, corrosion cast, murine renal cell carcinoma, vascular-disrupting agents, ZD6126 process in tumor growth and metastasis is now widely recognized and intensive research in recent years has resulted in a number of novel antiangiogenic agents (2). In adults, physiological angiogenesis is limited to a small number of specific processes, such as wound healing and renewal of the uterine lining (3). Targeting tumor angiogenesis has been shown to result in significant tumor growth delay in a wide range of animal models (4-7) and the clinical benefit of this approach has been confirmed in a number of studies (8-10).

Vascular-disrupting agents (VDAs) differ from antiangiogenic agents by targeting existing vasculature rather than preventing the growth of new vessels. The pivotal point for this therapeutic approach is the difference in the physiology of immature tumor vessels and mature normal vessels (11). A selective vascular disruption strategy is to target rapidly proliferating and immature tumor endothelial cells by interference with the tubulin cytoskeleton, which affects crucial endothelial cell function (12).

ZD6126 is a VDA which is rapidly converted in vivo by serum phosphatases to an active species, ZD6126 phenol (Nacetylcolchinol), which binds to tubulin, inhibiting tubulin polymerization, and thereby destabilizing the microtubule network (13). Proliferating endothelial cells, such as those of the tumor neovasculature, rely on the microtubule cytoskeleton to maintain their shape. The binding of ZD6126 phenol to tubulin causes rapid morphologic changes in the cell, leading to endothelial cell detachment, tumor blood vessel occlusion, and extensive tumor necrosis $(14,15)$. ZD6126 has been shown to exhibit significant antitumor activity in a broad range of in vivo animal tumor models (14). All VDAs evaluated to date produce a viable rim of surviving tissue at the periphery of the tumor, which is thought to arise because these cells can gain oxygen and nutrients from surrounding normal blood vessels $(14,16)$. Several Phase I studies in patients with advanced solid tumors have shown that, at tolerated doses, ZD6126 has antivascular effects in tumors $(17,18)$.

Biomarkers are important to establish biological activity and determine the optimal biological dose of novel therapies (19-21). In our study we investigated the influence of ZD6126 on tumor blood flow in afferent vessels to primary tumors by use of color Doppler imaging (CDI) ultrasound technique. 
The aim of this study was to determine the antitumor and antivascular activity of ZD6126 in the RENCA model and to evaluate its biological response using $\mathrm{CDI}$ as non-invasive biomarker. The orthotopic and syngeneic RENCA model is well characterized with respect to primary tumor formation in the kidney and the subsequent metastases formed in the lung (4). The influence of ZD6126 on the tumor blood vessel architecture was investigated by immunohistochemistry and by using a corrosion casting technique.

\section{Materials and methods}

Compounds. ZD6126 was provided by AstraZeneca (Macclesfield, UK)

Cell culture. Murine RENCA cells were originally obtained from a tumor that arose spontaneously in the kidney of BALB/c mice (22). Histologically, RENCA consists of granular cell-type adenocarcinoma, which is pleomorphic with large nuclei. Monolayers of murine RENCA cells were grown in RPMI-1640 with phenol red, supplemented with $10 \%$ fetal calf serum (FCS), 2 mM L-glutamine, 100 units $/ \mathrm{ml}$ penicillin, and $100 \mu \mathrm{g} / \mathrm{ml}$ of streptomycin. RENCA cells were cultured in a humidified atmosphere of $95 \%$ air and $5 \%$ carbon dioxide at $37^{\circ} \mathrm{C}$.

Mice. All experimental protocols were approved by the Ethics Committee for Animal Experimentation and performed according to the UK Coordinating Committee on Cancer Research Guidelines. The Experimental protocol is registered by the Regierungspräsidium Freiburg (reference number Az: 35-9185.82/3/216; Registrier-Nr. G-02/16). All experiments were performed on 6 weeks old female BALB/c mice (approximate weight, $18 \mathrm{~g}$; Charles River, Sulzfeld, Germany). Mice were anesthetized (1.5-2.5\% isoflurane with an oxygen flow of $21 / \mathrm{min})$ and injected with $0.2 \mathrm{ml}\left(4 \times 10^{6}\right)$ aliquots of RENCA cells in phosphate-buffered saline (PBS) into the subcapsular space of the left kidney through a flank incision. This model was first described by Murphy and colleagues (22) who showed that a subcapsular renal injection of RENCA cells in a syngeneic BALB/c mouse resulted in the progressive development of a primary tumor in the left kidney and spontaneous metastases to the regional lymph nodes, lung, peritoneum and liver (4). One week after cell implantation, the primary tumor is usually macroscopically visible; after $10 \mathrm{~d}$, spontaneous metastases may have developed in the regional lymph nodes, in the lung, the peritoneum, and the liver. The mean survival time of untreated RENCA-bearing mice is approximately $24 \mathrm{~d}$. Animal weights were taken every other day. Mice were sacrificed at pre-specified time points to determine weight and volume of primary tumor, weight and number of metastases of the lung, and metastasis formation in the abdominal lymph nodes. Animal weights were recorded three times a week and animal behavior and welfare were monitored daily.

Study design and treatments. ZD6126 was administered at a dose of $100 \mathrm{mg} / \mathrm{kg}$ by intraperitoneal (i.p.) injection. Day 0 was defined as time point when RENCA cells were injected into the left kidney. ZD6126 or vehicle were given on an intermittent schedule (day 10, 14 and 18; groups 1 and 2; n=15 per group) and animals sacrificed 1 day later (on day 22) (Fig. 1A). Following sacrifice assessments of primary tumors and metastases were performed and the efficacy of ZD6126 treat-ment compared with vehicle.

Color Doppler imaging study. To investigate the influences of ZD6126 on tumor blood flow in afferent vessels to primary tumors, color Doppler imaging ultrasound technique was used $(4,23)$. A single treatment of ZD6126 (100 mg/kg i.p.) or vehicle was performed at day 18 post cell injection (groups 3 and 4; ZD6126; each $\mathrm{n}=6$ ) in tumor-bearing (groups 3 and 4 ; each $\mathrm{n}=6$ ) and in age-matched non-tumor-bearing control animals (groups 5 and 6; each $n=6$ ) (Fig. 1B). Six hours after ZD6126 or vehicle administration primary tumors were located in all animals using a sonograph (Kranzbühler GE, Logic 700, München, Germany) with direct contact, high frequency ultrasound transducer. By color imaging, the left renal artery was detected Doppler measurements for systolic and diastolic blood flow and resistance index were performed. To rule out a systemic bias, comparable measurements were performed in the abdominal aorta.

Assessment of primary tumor and lung metastases. At the end of each study, mice were sacrificed and primary tumors or lungs were dissected and weighed. Primary tumor volume was assessed using calipers (length $\mathrm{x}$ width $\mathrm{x}$ diameter). Numbers of metastases in the lung were counted using a dissection microscope (Leica M-690, Germany; magnification $\mathrm{x} 14)$.

Histology. Healthy control kidneys and tumours were dissected immediately after death and weighed, photographed and cut in half with a razor blade. One half of the tissues were fixed in $4 \%$ paraformaldehyde buffered in $0.1 \mathrm{M}$ phosphate, and the other half was coated with tissue-tec mounting medium and frozen in liquid nitrogen for immunohistochemistry (see below). For determination of the tumor tissue fraction as well as for the assessment of necrosis, H\&E stained coronary sections through the middle of the tumor were photographed at low magnification with a Zeiss Axiophot photo microscope. Morphometry was carried out interactively using the Diskus 4.30 image analysis program (Hilgers, Königswinter). Hypochromatic areas as well as areas with pycnotic nuclei and disrupted cell borders were counted as necroses.

Immunohistochemistry. Tumor vasculature of primary tumors was assessed by two methods. In the intermittent dosing study, cryosections of the tissues (thickness $=5-10 \mu \mathrm{m}$ ) were taken from all animals. For the visualisation of blood vessels, immunohistochemical staining for CD31 (PECAM-1, MEC 13.3; PharMingen, San Diego, CA) was performed, and vessels were counted microscopically in vessel hot spots and scored as number of CD31 positive vessels per high power field using a defined magnification (x200). Alternatively, in the corrosion cast study (see below), cryosections ( $7 \mu \mathrm{m})$ were taken. Blood vessels were visualized using immunohistochemical staining for CD31 (MEC 13.3, PharMingen, San Diego, CA, USA). Vessel densities were determined at x100 magnification using a 25-point Chalkley grid, and values expressed as Chalkley points. 
Microvascular corrosion casting and morphometry. An additional study was performed to study the effects of ZD6126 on the tumor vasculature using quantitative three dimensional microvascular corrosion casting and to assess the extent of necrosis. On day 19 after tumor cell implantation, 60 tumorbearing animals were randomized to receive a single treatment with drug vehicle alone or with ZD6126 (100 mg/kg i.p.). At four time points ( $30 \mathrm{~min}, 2 \mathrm{~h}, 6 \mathrm{~h}, 24 \mathrm{~h}$ ) after ZD6126 administration animals in each group (groups 7-11; Fig. 1B) were sacrificed and prepared for analysis of tumor vessel architecture via electron microscopy using a corrosion cast technique, or tumors were excised and used for necrosis assessment and anti-CD31 staining. For corrosion casting, mice were anesthetized with $0.2 \mathrm{ml}$ i.p. Narcoren (Rhone Merieux, Laubheim, Germany). Blood clotting was prevented with 2000 IU i.p. Liquemin (Hoffmann-La Roche, Grenzach-Wyhlen, Germany). After thoracotomy, the heart and thoracic aorta were exposed. The left ventricle was punctured, an olive-tipped cannula was advanced through the aortic valve into the ascending aorta, and the vascular system was flushed with 10-15 ml of body warm saline. Vessel fixation was carried out with a $2.5 \%$ buffered glutaraldehyde solution in PBS (pH 7.40, 330 mosmol; Serva, Boehringer Ingelheim Bioproducts Partnership, Heidelberg, Germany). Thereafter, 10-15 $\mathrm{ml}$ of prepolymerized methyl methacrylate (Mercox CL-2B; Vilene Med, Tokyo, Japan), diluted with $20 \%$ methyl methacrylate monomers (Merck, Darmstadt, Germany), and catalyst was injected. The resin was allowed to cure for $2 \mathrm{~h}$ at $40^{\circ} \mathrm{C}$. Then, the tumor-bearing kidneys and contralateral healthy kidneys were excised, weighed and placed in $7 \% \mathrm{KOH}$ at $40^{\circ} \mathrm{C}$ to remove the surrounding tissue. The resin casts were washed in distilled water, frozen, cut into halves, dried, weighed, and mounted on aluminium stubs. The preparations were coated with gold (Balzers Union SCD 040 sputtercoater) and viewed with a scanning electron microscopy (Philips ESEM XL-30, Eindhoven, The Netherlands). Digital image overviews and stereopairs with a tilt angle of $6^{\circ}$ were taken for $3 \mathrm{D}$ image analysis.

3D-morphometry of the microvascular corrosion cast stereo images were performed on a KS300 (Kontron GmbH) image analysis system using own program routines for the parameters inter-vessel and inter-branch distances. Vessel diameters and variability of the mean vessel diameters were assessed with the Analysis 3.0 program.

Statistical analysis. All statistics were performed using the GraphPad Prism 5 (La Jolla, CA, USA) and SigmaPlot 11 (Systat Software Inc., San Jose, CA, USA) software package. All data are shown as means \pm SEM. The morphometric results obtained from the 3D microvascular corrosion casting images, are displayed as cumulative frequencies, allowing for better discrimination of individual percentiles. Statistical analyses of efficacy data were done using Student's t-test for unpaired groups. When normality tests failed, Mann Whitney rank sum test was used instead. A $\mathrm{P}<0.05$ was considered statistically significant.

\section{Results}

Antitumor activity of ZD6126. Previous studies have shown that the subcapsular renal injection of RENCA cells in
$\mathrm{BALB} / \mathrm{c}$ mice is followed by the progressive development of a primary tumor mass in the left kidney. Typically, 1 week after cell implantation, the primary tumor is macroscopically visible; after 10 days, spontaneous metastases may develop in the regional lymph nodes, lungs, peritoneum and liver. To determine whether ZD6126 is effective in inhibiting tumor growth in the RENCA model, mice were treated with repeated intermittent doses on day 10, 14 and 18 after RENCA tumor cells implantation into the lower pole of the left kidney. After repeated intermittent ZD6126 treatment (100 mg/kg i.p.) on day 10,14 , and 18 both the primary tumor volume and weight were significantly lower compared with controls (1.705 \pm $0.219 \mathrm{~cm}^{3}$ versus $2.999 \pm 0.334 \mathrm{~cm}^{3} ; \mathrm{p}<0.01$, and $0.768 \pm 0.131 \mathrm{~g}$ versus $2.244 \pm 0.325 \mathrm{~g} ; \mathrm{p}<0.01$, respectively) (Fig. 2).

ZD6126 was well tolerated in a dose of $100 \mathrm{mg} / \mathrm{kg}$ i.p. at intermittent application on day 10,14 and 18 and had no significant effects on body weight or the general well-being of the animals. However, although no weight loss was observed, administration of a single dose of ZD6126 (100 mg/kg i.p.) at day 19 post tumor cell implantation (i.e. animals had significant tumor burden at time of treatment) was not tolerated, causing diarrhoea and significant clinical deterioration, including some deaths, $24 \mathrm{~h}$ after ZD6126 administration. These adverse effects were associated of extensive drug-induced tumor edema within the kidney.

Effect of ZD6126 on metastasis. ZD6126 treatment did not significantly affect the number of lung metastases in any of the treatment schedules (data not shown). However, as previously reported (7), the number and sites of metastases showed high variability (data not shown) so the current experimental setting is unlikely to be sensitive enough to detect a treatment effect.

Effect of ZD6126 on vessel density. The vasculature from tumor tissues isolated from animals treated on day 10,14 and 18 post tumor cell implantation was stained for CD31. Vessel density in tumors from ZD6126-treated mice was higher than in tumors from vehicle treated controls (mean vessels per high power field $=87.39 \pm 5.049$ versus $60.25 \pm 3.608 ; \mathrm{p}<0.001$ ). Histological analysis showed a marked necrosis in ZD6126treated tumors associated with a viable rim of tumor cells (data not shown).

Blood flow changes under ZD6126 treatment. To evaluate the effect of ZD6126 on blood flow in afferent tumor vessels, the color Doppler imaging ultrasound technique was used. Eighteen days after tumor cell inoculation, primary tumors could be located by sonography in all animals. The resolution of the sonographic image allowed a distinction between the kidney, which showed hydronephrosis, and the primary tumor. Color imaging was used to locate the left renal artery, which could be detected reproducibly in all animals. The measurements of blood flow and resistance index in the left renal artery were performed by Doppler technique, resulting in a flow-time curve, which gives information on systolic blood flow $(\mathrm{cm} / \mathrm{s})$, diastolic blood flow $(\mathrm{cm} / \mathrm{s})$, resistance index, and heart rate (beats/min).

In the tumor feeding left renal artery, $6 \mathrm{~h}$ after treatment, a significant difference between control $(n=6)$ and ZD6126treated animals $(n=6)$ could be detected for systolic blood 
A

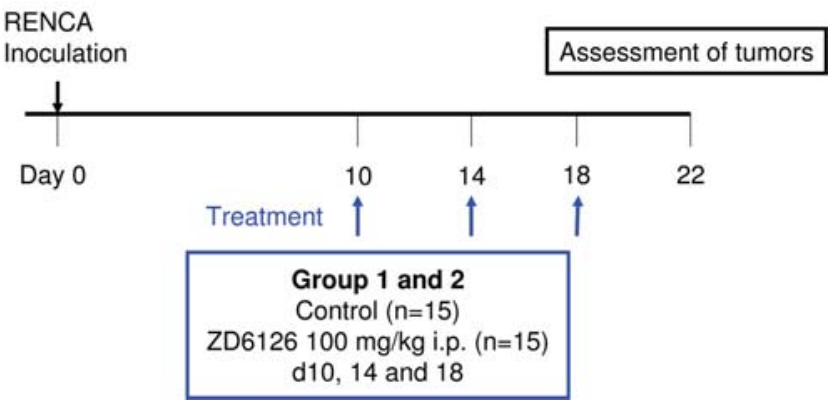

B

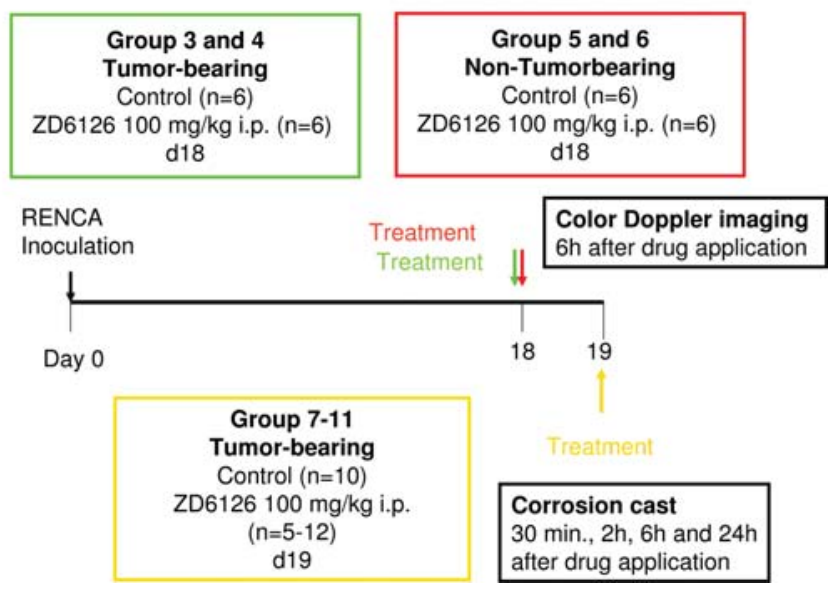

Figure 1. (A) Treatment schedule and time point for primary tumor assessment. Treatment on day 10,14 and 18. (B) Treatment schedule at day 18 for determination of blood flow using color Doppler imaging (CDI) in tumorbearing and non-tumor bearing mice (upper panel). Treatment schedule at day 19 for corrosion cast technique $30 \mathrm{~min}, 2 \mathrm{~h}, 6 \mathrm{~h}$, and $24 \mathrm{~h}$ after drug application (lower panel).

flow $(21.90 \pm 4.144 \mathrm{~cm} / \mathrm{s}$ versus $12.10 \pm 1.345 \mathrm{~cm} / \mathrm{s} ; \mathrm{p}=0.019)$, with a diastolic blood flow $(6.850 \pm 0.7377 \mathrm{~cm} / \mathrm{s}$ versus $3.633 \pm 1.064 \mathrm{~cm} / \mathrm{s} ; \mathrm{p}=0.0667$ ) (Fig. 3B). To rule out a systemic
Effect of ZD 6126 on primary tumor in a murine renal cell carcinoma

(Treatment days $d 10,14$ and 18 , animals sacrificed d 21)

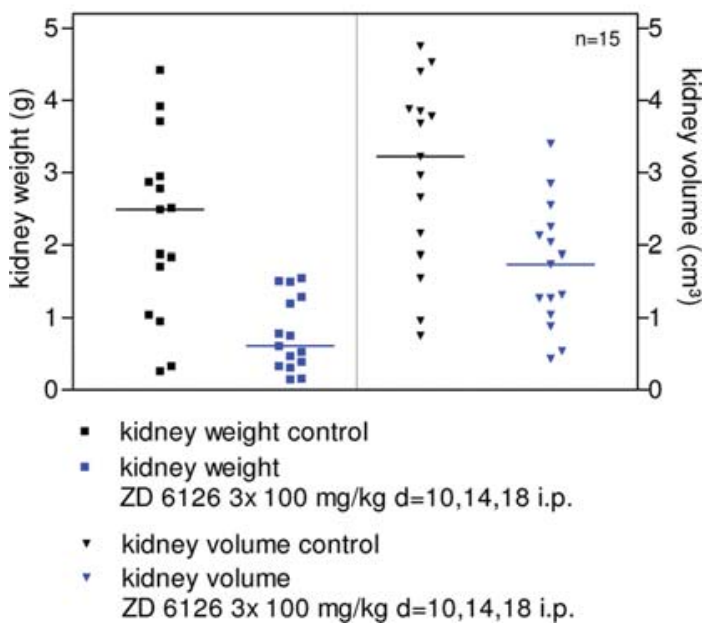

Figure 2. ZD6126 inhibits primary tumor growth in murine renal cell carcinoma. RENCA cells were inoculated into the subcapsular space of the left kidney of syngeneic BALB/c mice on day 0 . Mice were treated with ZD6126 (100 mg/kg i.p.) intermittent on day 10, 14, and 18. Primary kidney (tumor) weight and volume was assessed on day 21. Values are means; bars, SEM. ${ }^{* *} \mathrm{P}<0.01$ Mann-Whitney U test versus vehicle control.

bias, comparable measurements were performed in the abdominal aorta, where ZD6126-treated animals had a trend for reduced systolic and diastolic blood flow compared with control-treated animals, though this did not reach statistical significance (Fig. 3A). The resistance index and the heart rate were not affected by ZD6126 treatment (data not shown).

Corrosion cast study. In the corrosion cast study a single dose of ZD6126 (100 mg/kg i.p.) was given on day 19 after RENCA tumor cell implantation and animals sacrificed $30 \mathrm{~min}, 2 \mathrm{~h}, 6 \mathrm{~h}$ and $24 \mathrm{~h}$ after drug treatment, and compared with vehicle-
A

CDI of Aorta abdominalis

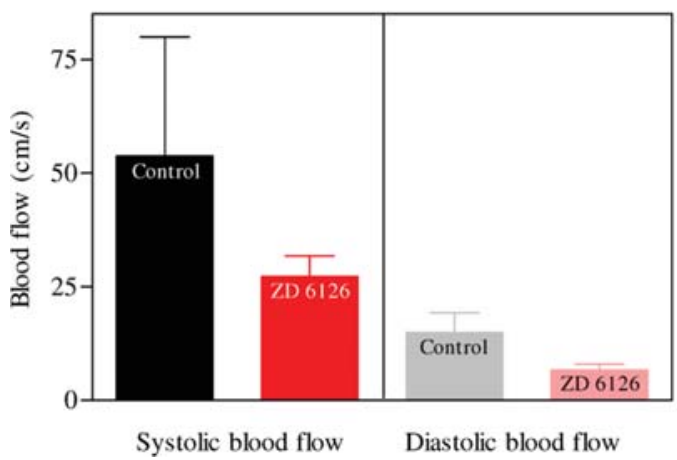

B

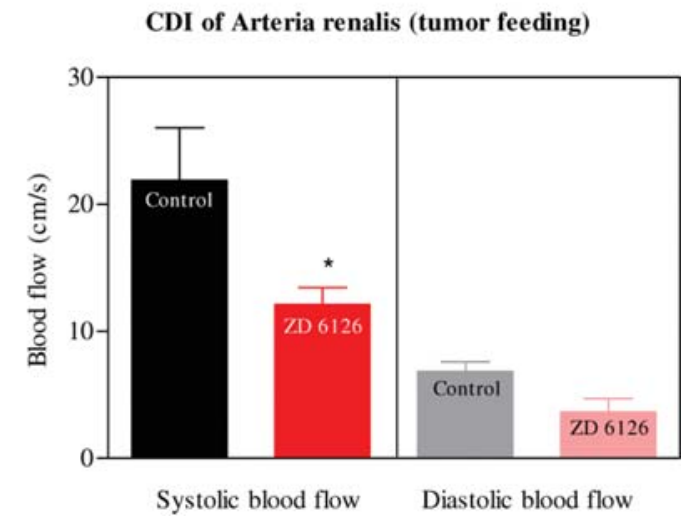

Figure 3. ZD6126 reduces blood flow in renal tumor artery. Effect of ZD6126 on blood flow in aorta abdominalis (A) and tumor-feeding renal artery (B) of murine renal cell carcinoma. Using the color Doppler imaging ultrasound technique, measurements of blood flow, 18 days after inoculation of RENCA cells, were performed. ZD6126 or vehicle were administered $6 \mathrm{~h}$ before examination. Values are means; bars, SE. Ps were calculated by comparing means of the treated group with means of the control group using the Mann Whitney t test. *Significant. 

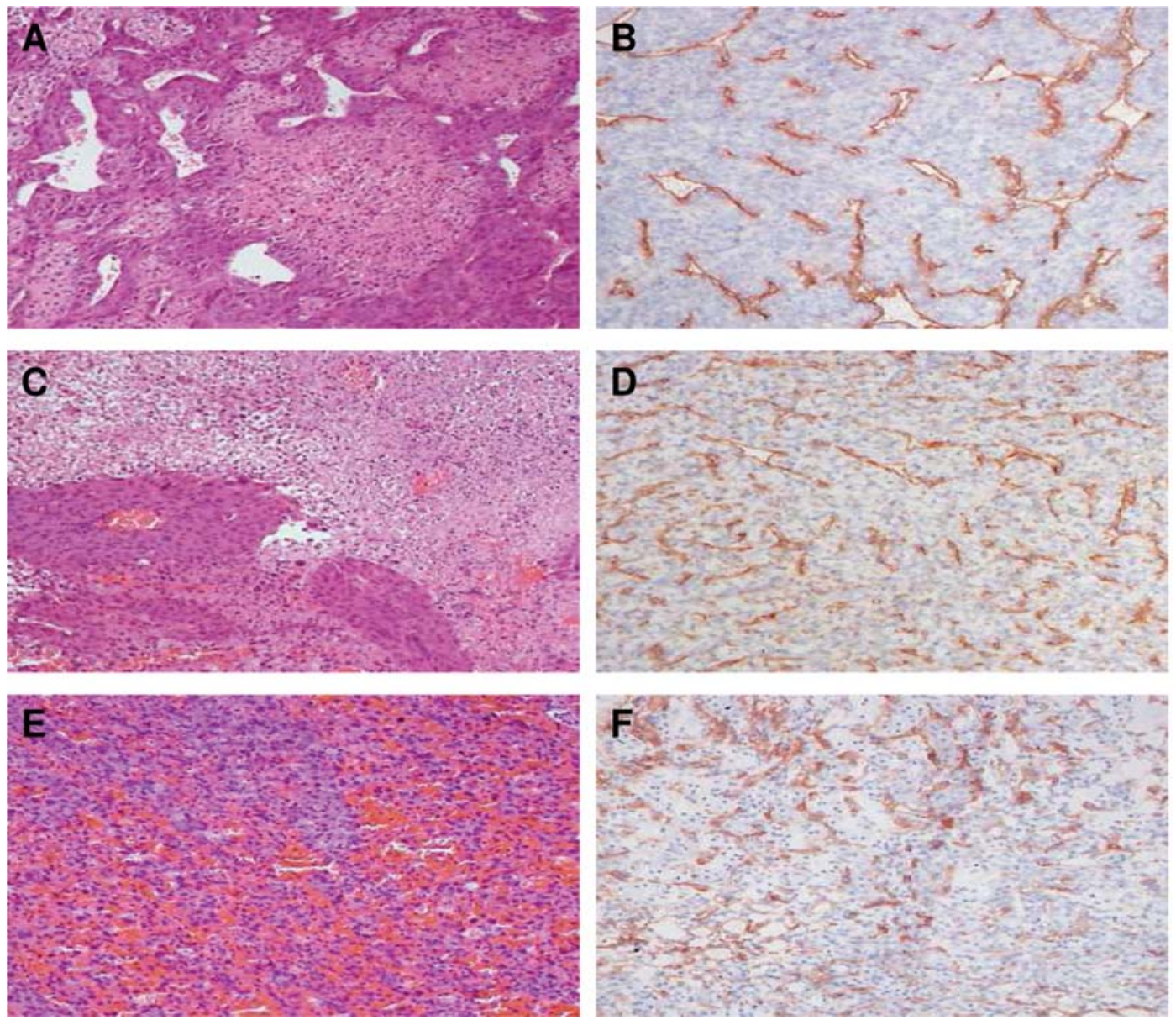

Figure 4. Light microscopy of untreated control tumors (A and B) demonstrating lacunary, sinusoidal vessels next to compressed vessels and a close side-byside of necrotic or hypochromatic areas and viable tumor cell masses. Thirty minutes after ZD6126 treatment (C and D) vessel diameters were heterogeneous and there was heterogeneous distribution of occluded vessels. Vessel occlusion did not result in immediate hypochromasia of the adjacent tumor cells (C). CD31 immunohistochemistry (D) stained occluded and compressed vessels. Twenty-four hours after ZD6126 treatment (E and F) there was widespread extravasation and vascular collapse. CD31 staining was present though it was more heterogeneous in intensity. A, C, E are H\&E stained; B, D, F are CD31 stained.

treated controls. Normal kidney and tumor tissue were isolated and evaluated by immunohistochemistry or transmission electron microscopy, or the microvasculature was evaluated by scanning electron microscopy of corrosion casts of normal and tumor-bearing kidney.

Following ZD6126 treatment, tumor weight was significantly decreased $30 \mathrm{~min}$ after ZD6126 treatment compared with controls $(1.686 \pm 0.326 \mathrm{~g}$ versus $2.908 \pm 0.352 \mathrm{~g} ; \mathrm{p}=0.02)$ whereas after $2 \mathrm{~h}, 6 \mathrm{~h}$ and $24 \mathrm{~h}$ tumor weight was not significantly different from to untreated tumors. We believe that the increase in tumor weight in the $2 \mathrm{~h}$ and $6 \mathrm{~h}$ groups as compared with the $30 \mathrm{~min}$ group was due, at least in part, to interstitial edema.

The percentage of necrosis versus viable tumor tissue was measured morphometrically in H\&E stained coronal sections by light microscopy (Fig. 4). Median necrotic fraction measurements in tumors ranged from 15.7 to $27.4 \%$, but there was no significant difference between the median necrosis scores in controls or any of the ZD6126 treatment groups.

The microvessel density was significantly decreased at $2 \mathrm{~h}$, $6 \mathrm{~h}$ and $24 \mathrm{~h}$, but not $30 \mathrm{~min}$ after ZD6126 treatment compared with control tumors (Figs. 4 and 5).

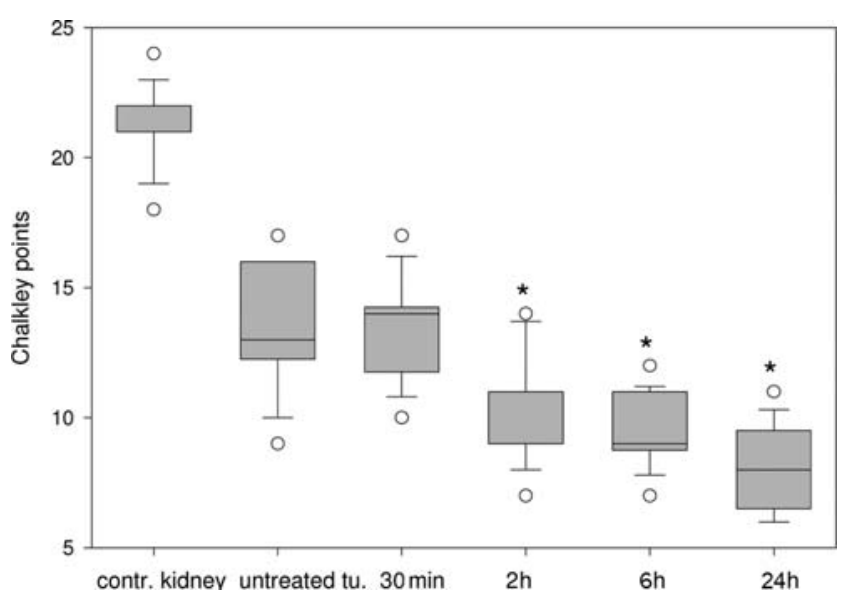

Figure 5. ZD6126 reduces microvessel density. RENCA cells were inoculated into the subcapsular space of the left kidney of syngeneic BALB/c mice on day 0. Mice were treated with ZD6126 (100 mg/kg i.p.) on day 19. At four time points ( $30 \mathrm{~min}, 2 \mathrm{~h}, 6 \mathrm{~h}$ and $24 \mathrm{~h}$ ) after ZD6126 administration animals in each group were sacrificed and staining for CD31 was performed. Values are expressed as Chalkley points. *Significant. 

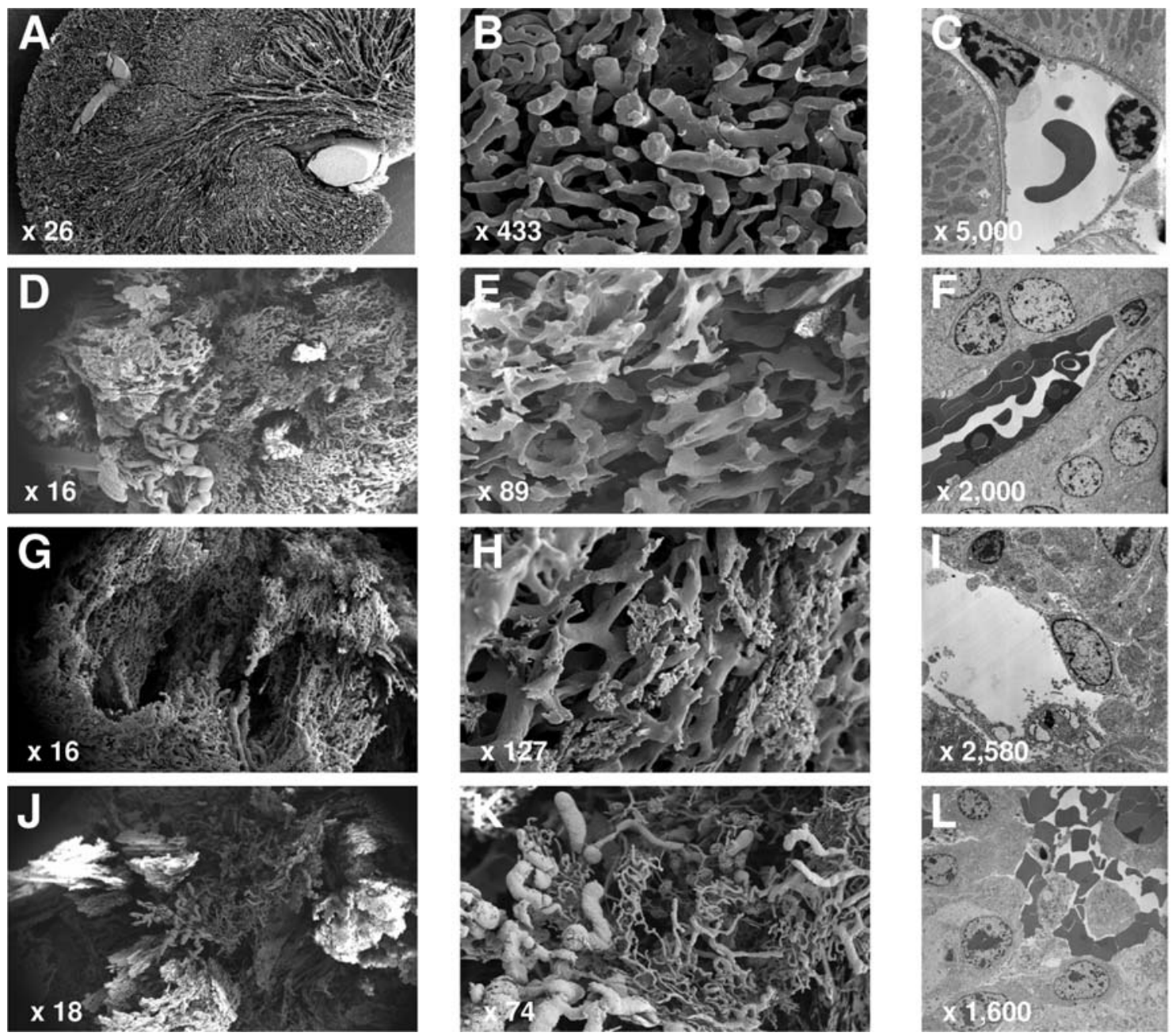

Figure 6. Scanning electron microscopy of corrosion casts of right control kidneys (A and B). The surface is covered by a terminal subcapsular plexus (c) with flatly extended, interwoven network topology. Transmission electron microscopy of control kidney tissue (C). Capillaries of the renal medulla are surrounded by a well demarcated basal lamina and pericytes are widely missing. The endothelium itself is comparatively low in height and in places luminal protrusions are seen. Untreated control tumor cast (D and E) with tortuous and dilated supplying and draining vessels. Numerous blind endings and bizarre vascular courses with varying vascular diameters are typical tumour vessel features. TEM images of control tumour without treatment (F) demonstrate a simple tumour vessel wall structure. Irrespective of the vessel diameter the vessel wall is mainly made up by a flattened endothelium although endothelial cell height is increased by intracellular edema and dilated endoplasmic reticulum. Clearly demarcated basal laminas are widely lacking, pericytes are not visible, and tumor cells line the endothelium. Thirty minutes after ZD6126 treatment (G, H and I) the vascular envelope is not continuously formed in the cast and some avascular or non-perfused areas are visible (G). In places, pre-existing vessels with regular courses and branching patterns are seen adjacent to tumor vessels with tiny extravasations $(\mathrm{H})$. Vessels with pronounced discontinuities are seen (I), with vacuolisation apparent. Twenty-four hours after ZD6126 treatment (J-L) the tumor centre shows the most pronounced heterogeneity in vessel densities (J). Pronounced vessel dilations and extravasations are seen only in tumor vessels, not in pre-existing host vessels (K). Transmission EM $24 \mathrm{~h}$ after treatment demonstrate heterogeneity in vessel morphology including vessels with severely disturbed endothelial cell morphology and disrupture (L). A, B, D, E, G, H, J, K scanning EM; C, F, I, L transmission EM.

Light microscopy of revealed typical tumour vessel structures. Both control and ZD6126 treated animals showed lacunary vessels next to compressed vessels and a close juxtaposition of necrotic or hypochromatic areas and viable tumour cell masses. Nearly all vessels had a capillary sinsusoidal wall lacking further differentiation. The strict hierarchy of the pre-existing vasculature was not visible and arteries and arterioles, frequently visible in the normal kidney tissue, were rarely found in the tumor mass.

More dilated, lacunary vessels were preferentially seen at the tumour periphery often next to autochthonous tissue. Adjacent to necrotic areas, broad rims of viable tumour cells with a width of 50-200 $\mu \mathrm{m}$ were arranged around patent vessels, indicative of the nutrient- and oxygen gradient from patent vessel to hypochromatic area. However, viable cell masses were also seen in areas with occluded vessels and increased extravasation. Semi-thin sections demonstrated a thin endothelium and frequently indications of vessel discontinuities (data not shown).

ZD6126 treatment resulted in an increase in extravasation and hemorrhages, which were most apparent $24 \mathrm{~h}$ after treatment. In general, a significant intratumoral and interindividual heterogeneity of vessel occlusion was evident. ZD6126induced loss of endothelial cell integrity was not clearly apparent at early time points ( $\leq 2 \mathrm{~h}$ ). However, 6 and $24 \mathrm{~h}$ after ZD6126 treatment, extravasations and vessel collapse was pronounced and accompanied by a higher degree of tumor cell and nuclei polymorphism and, in places, apoptotic 


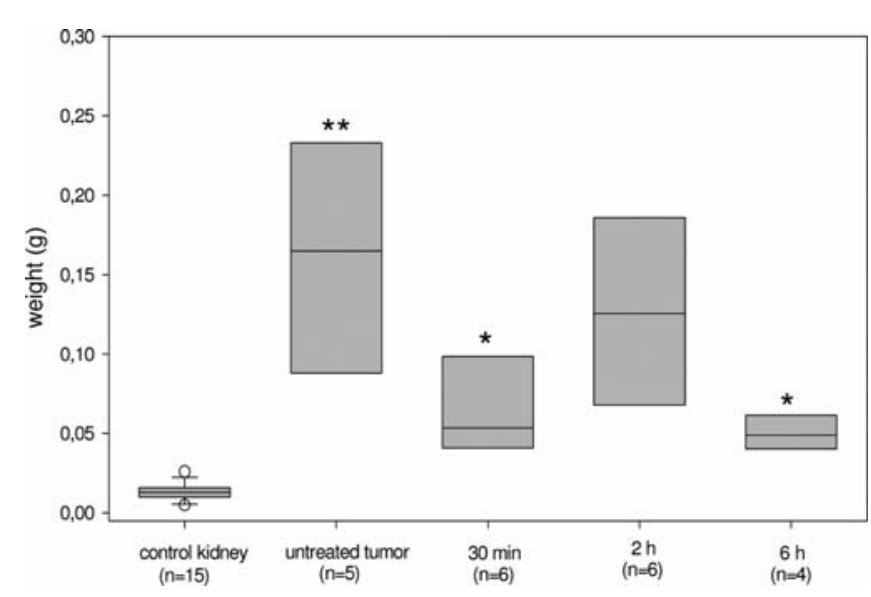

Figure 7. Parameters of vessel architecture assessed in corrosion casts. Vascular volume assessed with cast weight. From the specimen weights after perfusion and the weights of the corroded specimens the vascular volumes were calculated. * Significant between untreated tumors and $30 \mathrm{~min}(\mathrm{p}=0.049)$ and $6 \mathrm{~h}(\mathrm{p}=0.040) .{ }^{* *}$ Significant between control kidneys and untreated tumors $(\mathrm{p}=0.001)$.

figures. All the histological phenomena described were present in all treatment groups, including controls, though to differing extents.

Transmission electron microscopy was able to examine the vessel wall in more detail (Fig. 6). Compared with capillary vessels of the renal medulla in normal kidney, which are always surrounded by a well demarcated basal lamina, the tumor vessels did not consistently form basal lamina. Instead, a direct contact between tumor cells and endothelial cells was usually visible. Apart from some discontinuities in altered vessels, and irrespective of diameter, the tumor vessels generally had a flattened endothelium without fenestrations whereas the control tissue vessels are characterised by continuous fenestrated endothelium.

Early (30 min) after ZD6126 treatment, capillaries with increased vacuolization (possibly hypoxia-related) and changes in cell morphology (possibly related to cytoskeleton alterations) were noted. However, endothelial cell integrity appeared to be retained. In the later treatment groups $(\geq 2 \mathrm{~h})$, intracellular edema, vacuolization and membrane damage increased. Occasional endothelial cells appeared to be rounded-up although this was not always associated with a loss of endothelial coverage. Six and $24 \mathrm{~h}$ after ZD6126 treatment, more severe focal endothelial cell damage with cell membrane rupture, edema, and haemorrhage was visible.

Scanning electron microscopy of microvascular corrosion casts of normal kidney displayed the typical architecture: the subcapsular plexus with horizontally orientated vessels fed from ascending branches of the interlobular arteries, which branch off in the cortex - medulla zone (Fig. 6). The kidney medulla is characterized by mainly parallel orientated vessels next to the collecting ducts and ascending and descending limbs of the loop of Henle. In the cortex, numerous vessels feed the glomerula.

In the tumor casts, however, organ specific vascularity is seen only rarely. RENCA tumor growth was accompanied by the formation of a vascular network with properties of

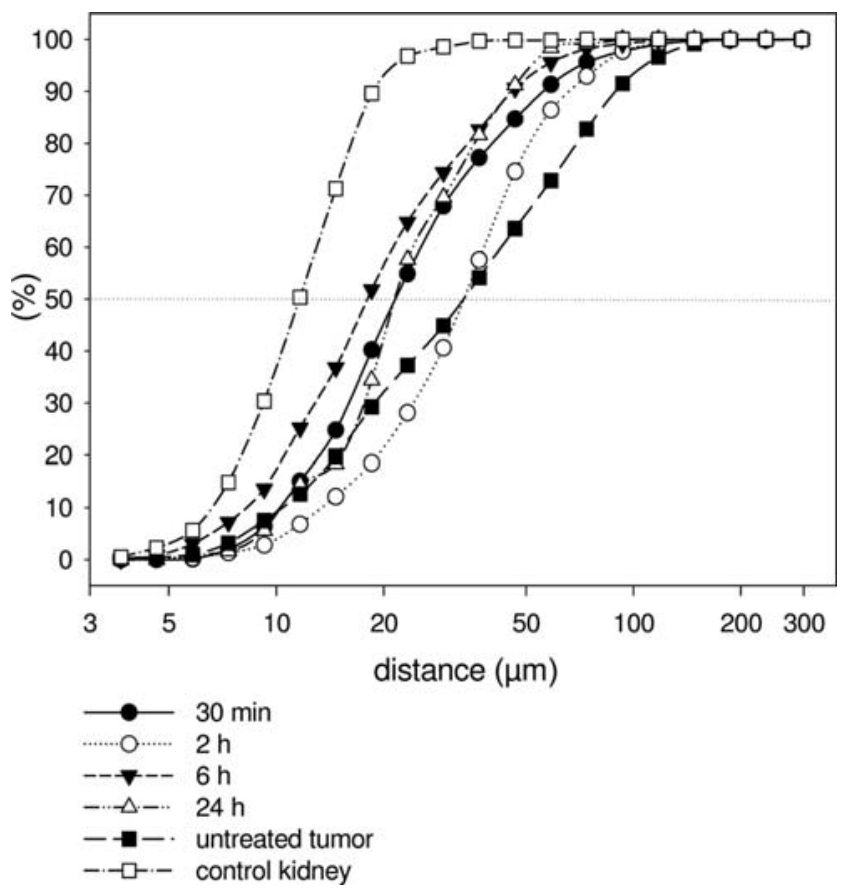

Figure 8. Parameters of vessel architecture assessed in corrosion casts: intervessel distances.

characteristic of tumors: loss of hierarchy, flattening of main vessels, avascular areas next to vascular hot spots, capillary elongations and blind ends. The periphery of the tumor had the highest vessel densities. The vessel diameters vary considerably and clearly demarcated arteries were seen only rarely.

ZD6126 treatment increased extravasation which was most apparent $24 \mathrm{~h}$ after treatment. Vessel shut down, determined by the increase in total avascular (non-perfused) area, increased up to $24 \mathrm{~h}$ after ZD6126 treatment.

\section{Tumor vessel morphometry}

Vascular volumes. The weights of the corrosion cast specimens (as a measure of the vascular volumes) were calculated. The vascular cast weight $30 \mathrm{~min}$ and $6 \mathrm{~h}$ after ZD6126 treatment was significant lower than in untreated tumors (Fig. 7). However, the corrosion casts represent only perfused vasculature regions; occluded vessels would not be permeated by the vascular casting agent. In addition, interpretation is further complicated because extravasated casting medium, especially $6 \mathrm{~h}$ and $24 \mathrm{~h}$ after ZD6126 treatment, would not represent the vascular volume but would contribute to the cast weight.

\section{Vessel measurements}

Inter-vessel distances. Inter-branch distances (representing the length of individual vessel segments) and vessel diameters determined by three-dimensional morphometry of the corrosion casts are shown as cumulative frequency plots (Figs. 8-10). The lowest values for each of these vessel measurements are found in normal kidney, and the highest in untreated tumor samples. Generally, vessel measurements for ZD6126-treated samples were significantly lower than in untreated tumors $(\mathrm{P}<0.01)$ and significantly higher than in control kidney 

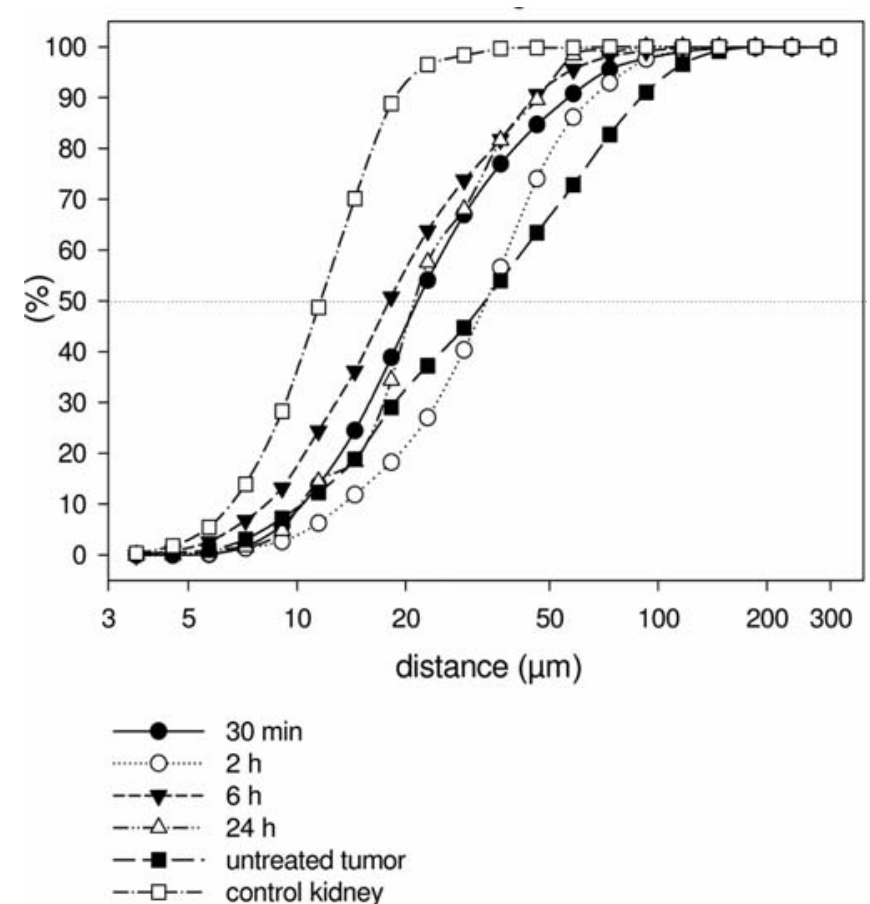

Figure 9. Parameters of vessel architecture assessed in corrosion casts: interbranch distances.

$(\mathrm{P}<0.01)$. An exception was seen for tumor samples taken $2 \mathrm{~h}$ post-ZD6126 treatment, where vessel measurements were not different from untreated tumors, although at least for intervessel distance and inter-branch distance, the skewedness of the data decreased.

\section{Discussion}

Recent research in the treatment of cancer has focused on seeking novel strategies to control cell growth and metastasis. One such strategy is to target angiogenesis, and such treatment primarily exerts a cytostatic action, thereby preventing further tumor vascularization, slowing primary tumor growth and limiting the development of metastases (24).

The aim of our study was to investigate the antitumor and antiangiogenic effects of ZD6126, a highly potent and selective tumor VDA. Treatment with ZD6126 led to a significant reduction of primary tumor growth in the murine RENCA model. The most significant effect was observed for intermittent treatment with ZD6126 on day 10, 14 and 18. ZD6126 was generally well tolerated in RENCA mice and did not cause decrease in animal body weights related to the control animals. However, treatment with ZD6126 was not well tolerated when used to treat mice with a large tumor burden ( $\geq$ day 18) where drug treatment appeared to induce significant tumor edema. The vessel density in primary tumors was significantly increased following ZD6126 treatment, possibly due to induced tumor hypoxia with a compensatory increase of microvessels. ZD6126 caused extensive necrosis in the central regions of tumors, but in the tumor periphery a rim of viable tumor tissue remains.

These data confirm the antitumor potential of ZD6126, seen in other tumor models $(14,25-27)$. In a study by Blakey

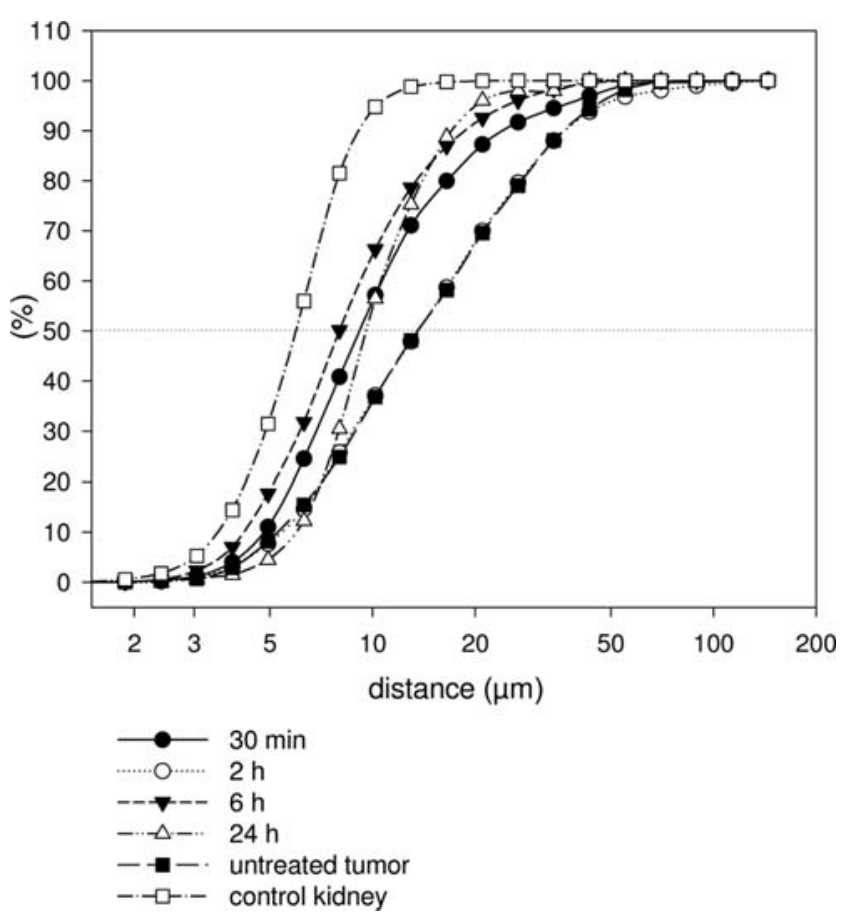

Figure 10. Parameters of vessel architecture assessed in corrosion casts: vessel diameters.

et al the antitumor effects of ZD6126 was examined in tumor xenografts in nude mice from human lung, colorectal, prostate, ovarian and breast carcinoma (14). Treatment with ZD6126 led to extensive tumor necrosis $24 \mathrm{~h}$ after administration, and the combination with cisplatin enhanced the tumor growth delay. In dose-response studies these effects were seen at a dose 8- to 16-fold lower than the maximum tolerated dose.

A major obstacle in the development of targeted agents, particularly those inhibiting angiogenesis, is the lack of noninvasive biomarkers to monitor response during treatment. In contrast to identifying the maximal tolerable dose, determination of the optimal biological dose, reaching biological activity at lower doses, has become the main target in the early development of anti-angiogenic agents. This has been evaluated by different biomarker techniques (19). As a new standard in anti-tumor treatment, a better understanding of imaging in the treatment monitoring for anti-angiogenic agents is important. In the present study, CDI was used as biomarker to measure blood flow in the tumor under treatment. Ultrasound is a favored modality for imaging angiogenesis because it does not expose patients to any significant risks, it is widely available, it can be repeated often, and it is mobile (19). In a study with PTK787/ZK 222584, a VEGF receptor tyrosine kinase inhibitor, CDI was used as surrogate marker in preclinical and clinical studies (4). In the RENCA mouse model, PTK/ZK reduced systolic tumor blood flow by $44 \%$ (4). In our study we found a significant reduction of systolic blood flow in the tumor feeding left arteria renalis in the ZD6126-treated animals compared to vehicle controls. The blood flow was decreased by $55 \%$ as compared with untreated animals. Resistance index and heart rate were not affected. Thus CDI may be used to non-invasively measure efficacy of ZD6126 treatment. 
Detailed knowledge of tumor vascularization can determine blood flow, tumor biology, and the efficacy of therapeutic agents. To this end, corrosion casting technology was also used in order to visualize the vessel architecture of primary tumors three dimensionally $(28,29)$. In this study we did not see an increase in necrosis at the single treatment on day 19 even though the vessel densities decreased. Davis et al (13) found an induction of necrosis with increases of the median necrosis index from 1.5 to 5.5 with low doses and 9 with high doses. However, they used the comparatively slow growing CaNT with diameters of 5-6 mm. Our fast growing, untreated RENCA tumors had a median weight of $3.3 \mathrm{~g}$, significantly higher than in the previous study. Apart from the differences in size the CaNT tumors were transplanted subcutaneously, a site with more limited blood flow and preexisting vessels flow compared with parenchymal organs. In contrast, in the RENCA-model the tumor is transplanted into a fascia- and capsule defined region with very high blood flow (renal blood flow: 10\% of heart minute volume per kidney). Blood flow and number of pre-existing vessels is, thus, at least 20 times higher than in a subcutaneous area of the same volume. This might also explain for the comparatively low necrosis fraction in the untreated tumors with disseminated necrotic areas. Single therapy at day 19 did not result in the formation of a large central necrosis, as was seen in murine subcutaneous tumors: this can be explained in terms of a better vascular supply in the RENCA-model with broad arterial supply. In addition, we would not expect a starvation induced necrosis in less than $24 \mathrm{~h}$, if the pre-therapeutic $\mathrm{pO}^{2}$ was higher than 5-10 $\mathrm{mmHg}$. This may in part explain the lack of significant changes in tumor weight in animals measured $2 \mathrm{~h}, 6 \mathrm{~h}$ and $24 \mathrm{~h}$ after ZD6126 treatment. In addition, intracellular and interstitial edema formation as well as the short time period after single dose ZD6126 treatment makes significant decrease in tumor sizes unlikely.

We found a decrease in the vessel densities in highly vascularized areas (hot spots) in animals treated at day 19 with ZD6126. More dramatic decreases in perfusion were recorded by Siemann et al (15). They reported decreases from a count of 47 to 15 after $24 \mathrm{~h}$. However, they assessed with perfusion of Hoechst 33342 dye, a marker of perfused vessels, whereas we counted the total number of CD31stained vessels irrespective of their functionality, or integrity.

Both light and scanning transmission electron microscopy showed ZD6126-induced increases in hemorrhage and extravasation, as previously reported by Goto et al (25). The functional-structural correlate for this is damage of endothelial cells. All the above-mentioned papers ascribe the endothelial cell damage to the tubulin destabilizing properties of ZD6126, which would be expected to result in a perinuclear rounding-up of the endothelial cells, exposure of the basal lamina after retraction of endothelial cells, and subsequent thrombosis and/or vessel congestion. However, apart from the extrapolation of in vitro experiments showing cytoskeletal changes to the in vivo situation, and vessel congestion there is little experimental evidence for the proposed sequence of events in this attractive concept.

Congestion of numerous large caliber vessels, as demonstrated in light microscopy, make luminal diameter restrictions in these vessels as primary cause for blood flow cessation unlikely. Here we believe that an obstruction or damage of the efferent draining vessels may induce blood flow cessation in lacunary sinusoidal vessels. This implies that tumor endothelial cells do not react uniformly to the agent.

Our transmission electron microscopic studies showed only occasional evidence of the proposed rounding phenomena for endothelial cells and no clear evidence of retraction of endothelial cell linings with denudation of the basal lamina. Also, we saw only rarely thrombocyte degranulation or thrombus formation, but, instead, simple vessel congestion. The endothelial cells showed in most cases lytic damage of the cell membrane without cell retraction phenomena and without perinuclear rounding.

Our data do not necessarily contradict previous studies: tumor vessels of different origin may, in structural terms, be comparable. In our model, the typical structural characteristics of tumor vasculature were seen. However, when comparing tumor vessels with normal tissue vessels significant differences exist.

Using scanning electron microscopy of corrosion casts we found ZD6126 induced decreases in vessel diameter. Congested or thrombotic vessels are not represented since they cannot be filled with the casting medium. If the remaining vessel diameter had decreased by endothelial cell rounding-up, this would have been visible in semi-thin sections and transmission electron microscopy. Therefore, we regard the shift in vessel diameter as a result of a proportional increase of pre-existing small caliber vessels. That the latter are not affected by the agent, as reported by Davis et al (13), holds true also for our experimental setting. This seems to be also the reason for the changes in intervessel and inter-branch distances. If the agent shutted down vessels without focal preferences, the effect would have been even bigger.

In conclusion, vascular tumor targeting with ZD6126 significantly inhibited primary tumor growth in this clinically relevant, orthotopic RENCA model. ZD6126 caused tumor necrosis and this is possibly due to a direct action of ZD6126 on tumor endothelium. Changes of tumor blood flow measured by CDI may demonstrate biological activity of ZD6126. Tumor vessel architecture and morphometry was influenced by ZD6126-treatment.

\section{Acknowledgements}

A.J. Ryan is an AstraZeneca employee. The authors have no conflict of interests to declare.

\section{References}

1. Folkman J: Tumor angiogenesis: therapeutic implications. N Engl J Med 285: 1182-1186, 1971.

2. Medinger $M$ and Drevs $\mathrm{J}$ : Receptor tyrosine kinases and anticancer therapy. Curr Pharm Des 11: 1139-1149, 2005.

3. Folkman J: Angiogenesis in cancer, vascular, rheumatoid and other disease. Nat Med 1: 27-31, 1995.

4. Drevs J, Hofmann I, Hugenschmidt H, Wittig C, Madjar H, Muller M, Wood J, Martiny-Baron G, Unger C and Marme D: Effects of PTK787/ZK 222584, a specific inhibitor of vascular endothelial growth factor receptor tyrosine kinases, on primary tumor, metastasis, vessel density, and blood flow in a murine renal cell carcinoma model. Cancer Res 60: 4819-4824, 2000. 
5. Drevs J, Konerding MA, Wolloscheck T, Wedge SR, Ryan AJ, Ogilvie DJ and Esser N: The VEGF receptor tyrosine kinase inhibitor, ZD6474, inhibits angiogenesis and affects microvascular architecture within an orthotopically implanted renal cell carcinoma. Angiogenesis 7: 347-354, 2004.

6. Kim KJ, Li B, Winer J, Armanini M, Gillett N, Phillips HS and Ferrara N: Inhibition of vascular endothelial growth factorinduced angiogenesis suppresses tumor growth in vivo. Nature 362: 841-844, 1993

7. Medinger M, Esser N, Zirrgiebel U, Ryan A, Jürgensmeier JM and Drevs J: Antitumor and antiangiogenic activity of cediranib in a preclinical model of renal cell carcinoma. Anticancer Res 29: 5065-5076, 2009.

8. Escudier B, Eisen T, Stadler WM, Szczylik C, Oudard S, Siebels M, Negrier S, Chevreau C, Solska E, Desai AA, Rolland F, Demkow T, Hutson TE, Gore M, Freeman S, Schwartz B, Shan M, Simantov R and Bukowski RM: Target Study Group: sorafenib in advanced clear-cell renal-cell carcinoma. N Engl J Med 356: 125-134, 2007.

9. Motzer RJ, Hutson TE, Tomczak P, Michaelson MD, Bukowski RM, Rixe O, Oudard S, Negrier S, Szczylik C, Kim ST, Chen I, Bycott PW, Baum CM and Figlin RA Sunitinib versus interferon alpha in metastatic renal-cell carcinoma. N Engl J Med 356: 115-124, 2007.

10. Hurwitz H, Fehrenbacher L, Novotny W, Cartwright T, Hainsworth J, Heim W, Berlin J, Baron A, Griffing S, Holmgren E, Ferrara N, Fyfe G, Rogers B, Ross R and Kabbinavar F: Bevacizumab plus irinotecan, fluorouracil, and leucovorin for metastatic colorectal cancer. N Engl J Med 350: 2335-2342, 2004.

11. Thorpe PE: Vascular targeting agents as cancer therapeutics. Clin Cancer Res 10: 415-427, 2004.

12. Tozer GM, Kanthou C and Baguley BC: Disrupting tumor blood vessels. Nat Rev Cancer 5: 423-435, 2005.

13. Davis PD, Dougherty GJ, Blakey DC, Galbraith SM, Tozer GM, Holder AL, Naylor MA, Nolan J, Stratford MR, Chaplin DJ and Hill SA: ZD6126: a novel vascular-targeting agent that causes selective destruction of tumor vasculature. Cancer Res 62: 7247-7253, 2002.

14. Blakey DC, Westwood FR, Walker M, Hughes GD, Davis PD, Ashton SE and Ryan AJ: Antitumor activity of the novel vascular targeting agent ZD6126 in a panel of tumor models. Clin Cancer Res 8: 1974-1983, 2002

15. Siemann DW and Rojiani AM: Enhancement of radiation therapy by the novel vascular targeting agent ZD6126. Int J Radiat Oncol Biol Phys 53: 164-171, 2002.

16. Evelhoch JL, LoRusso PM, He Z, DelProposto Z, Polin L, Corbett TH, Langmuir P, Wheeler C, Stone A, Leadbetter J, Ryan AJ, Blakey DC and Waterton JC: Magnetic resonance imaging measurements of the response of murine and human tumors to the vascular-targeting agent ZD6126. Clin Cancer Res 10: 3650-3657, 2004.

17. Beerepoot LV, Radema SA, Witteveen EO, Thomas T, Wheeler C, Kempin S and Voest EE: Phase I clinical evaluation of weekly administration of the novel vascular-targeting agent, ZD6126, in patients with solid tumors. J Clin Oncol 24: 1491-1498, 2006.
18. LoRusso PM, Gadgeel SM, Wozniak A, Barge AJ, Jones HK, DelProposto ZS, DeLuca PA, Evelhoch JL, Boerner SA and Wheeler C: Phase I clinical evaluation of ZD6126, a novel vascular-targeting agent, in patients with solid tumors. Invest New Drugs 26: 159-167, 2008.

19. Drevs J and Schneider V: The use of vascular biomarkers and imaging studies in the early clinical development of anti-tumor agents targeting angiogenesis. J Intern Med 260: 517-529, 2006.

20. Drevs J, Siegert P, Medinger M, Mross K, Strecker R, Zirrgiebel U, Harder J, Blum H, Robertson J, Jürgensmeier JM, Puchalski TA, Young H, Saunders O and Unger C: Phase I clinical study of AZD2171, an oral vascular endothelial growth factor signaling inhibitor, in patients with advanced solid tumors. J Clin Oncol 25: 3045-3054, 2007.

21. Drevs J, Medinger M, Mross K, Fuxius S, Hennig J, Buechert M, Thomas A, Unger C, Chen BL, Lebwohl D and Laurent D: A phase IA, open-label, dose-escalating study of PTK787/ZK 222584 administered orally on a continuous dosing schedule in patients with advanced cancer. Anticancer Res 30: 2335-2339, 2010.

22. Murphy GP and Hrushesky WJ: A murine renal cell carcinoma. J Natl Cancer Inst 50: 1013-1025, 1973.

23. Goertz DE, Yu JL, Kerbel RS, Burns PN and Foster FS: Highfrequency Doppler ultrasound monitors the effects of antivascular therapy on tumor blood flow. Cancer Res 62: 6371-6375, 2002.

24. Carmeliet P: Angiogenesis in health and disease. Nat Med 9: 653-660, 2003

25. Goto H, Yano S, Zhang H, Matsumori Y, Ogawa H, Blakey DC and Sone S: Activity of a new vascular targeting agent, ZD6126, in pulmonary metastases by human lungadenocarcinoma in nude mice. Cancer Res 62: 3711-3715, 2002.

26. Horsman MR and Murata R: Vascular targeting effects of ZD6126 in a C3H mouse mammary carcinoma and the enhancement of radiation response. Int J Radiat Oncol Biol Phys 57: 1047-1055, 2003

27. Kleespies A, Köhl G, Friedrich M, Ryan AJ, Barge A, Jauch KW and Bruns CJ: Vascular targeting in pancreatic cancer: the novel tubulin-binding agent ZD6126 reveals antitumor activity in primary and metastatic tumor models. Neoplasia 7: 957-966, 2005.

28. Konerding MA, Fait E and Gaumann A: 3D microvascular architecture of pre-cancerous lesions and invasive carcinomas of the colon. Br J Cancer 84: 1354-1362, 2001.

29. Konerding MA, Malkusch W, Klapthor B, et al: Evidence for characteristic vascular patterns in solid tumors: quantitative studies using corrosion casts. Br J Cancer 80: 724-732, 1999. 